Check for updates

Cite this: RSC Adv., 2019, 9, 20931

Received 11th April 2019

Accepted 11th June 2019

DOI: $10.1039 / c 9 r a 02730 k$

rsc.li/rsc-advances

\section{Organic-inorganic hybrid perovskite quantum dot light-emitting diodes using a graphene electrode and modified PEDOT:PSS $\uparrow$}

\author{
Qing Zhang, ${ }^{a}$ Hongtao $\mathrm{Yu}{ }^{a}$ Ziwei Liu, ${ }^{a}$ Yao Lu, ${ }^{\mathrm{a}}$ Danqing $\mathrm{Ye},{ }^{\mathrm{a}}$ Jie Qian, ${ }^{\mathrm{a}}$ Yanan $\mathrm{Wu},{ }^{\mathrm{a}}$ \\ Wenwen Gu, ${ }^{a}$ Ben Ma, ${ }^{a}$ Liuquan Zhang, ${ }^{a}$ Yu Duan, ID ${ }^{b}$ Lihui Liu, ${ }^{a}$ Kun Cao, ${ }^{a}$ \\ Shufen Chen (iD *ac and Wei Huang iD *ac
}

Perovskite quantum dot (PQD) light-emitting diodes (LEDs) have rapidly developed in the past several years due to the excellent optoelectronic properties of lead halide perovskites. However, PQD LEDs using graphene electrodes have not been reported despite their huge potential for applications in flexible displays and lighting sources. Herein, graphene was first used as the electrode of PQD LEDs. To overcome graphene's limitations such as hydrophobicity and graphene-induced film nonuniformity, the modification of poly(3,4-ethylenedioxythiophene):poly(styrenesulfonate) (PEDOT:PSS) with Triton X-100 and dimethyl sulfoxide (DMSO) codoping was reported, which not only improved the wettability of the graphene surface and the sequent film quality, but also reduced the dissolution of the PQD solvent to the bottom poly[ $N, N^{\prime}$-bis(4-butylphenyl)- $N, N^{\prime}$-bis(phenyl)-benzidine] and PEDOT:PSS. More importantly, the synergistic effect of Triton X-100 and DMSO altered the PEDOT:PSS morphology from a coiled structure to a nanofibril conductive network, sufficiently enhancing the electrical conductivity of PEDOT:PSS. With this modification strategy, green PQD LEDs with $\mathrm{CH}_{3} \mathrm{NH}_{3} \mathrm{PbBr}_{3}$ emission layers were successfully fabricated on graphene anodes, with 3.7- and 4.4-fold enhancements in luminance and current efficiency, respectively, compared to those of their counterparts without PEDOT:PSS modification. The film modification strategy and graphene-based PQD LEDs in this work are expected to shed light on the further design and manufacture of flexible highly efficient PQD display and lighting devices.

\section{Introduction}

Perovskite quantum dot (PQD) light-emitting diodes (LEDs) have rapidly developed in the past several years due to the excellent optoelectronic properties of lead halide perovskites including facile tunable luminance over the entire visible spectrum, a high photoluminescence quantum yield (PLQY) of up to $100 \%$ and narrow full width at half maximum (FWHM). ${ }^{1-15}$ By using the strategy of anion exchange and quantum confinement effect of these PQDs, an ultrawide color gamut of $>120$ is achieved in PQD LEDs, exceeding that of organic light-

${ }^{a}$ Key Laboratory for Organic Electronics and Information Displays \& Jiangsu Key Laboratory for Biosensors, Institute of Advanced Materials (IAM), Jiangsu National Synergetic Innovation Center for Advanced Materials (SICAM), Nanjing University of Posts \& Telecommunications (NUPT), 9 Wenyuan Road, Nanjing 210023, China. E-mail: iamsfchen@njupt.edu.cn; iamdirector@fudan.edu.cn

${ }^{b}$ State Key Laboratory on Integrated Optoelectronics, College of Electronic Science and Engineering, Jilin University, Changchun 130012, China

'Institute of Flexible Electronics (SIFE), Northwestern Polytechnical University (NPU), 127 West Youyi Road, Xi'an 710072, Shaanxi, China. E-mail: provost@nwpu.edu.cn

$\dagger$ Electronic supplementary information (ESI) available. See DOI: 10.1039/c9ra02730k emitting diodes (OLEDs), bulk perovskite light-emitting diodes (PeLEDs) and Cd/Zn-based quantum dot LEDs. ${ }^{16}$ These obvious advantages endow PQD LEDs with a huge potential for applications in next-generation high-definition displays and high-quality lighting sources.

ITO has been widely used as the electrode of OLEDs, organic solar cells (OSCs) and perovskite photovoltaic devices due to its high intrinsic transmissivity and good conductivity. However, the brittle nature makes it unsuitable for future applications in flexible optoelectronic devices. ${ }^{17-19}$ Moreover, indium ion diffusion into the bottom transport layer triggers the degradation of a device's performance and lifetime..$^{20}$ Therefore, the development of new transparent conductive materials to replace ITO is considered as a major resolution strategy. Representative flexible electrodes containing conducting polymers, ${ }^{21,22}$ carbon nanotubes, ${ }^{23,24}$ graphene, ${ }^{25,26}$ and metal nanowires ${ }^{27,28}$ have been extensively studied in the past decade. Among them, metal nanowires and graphene flexible electrodes have shown excellent optical and electrical performances in flexible OLED and OSC applications owing to their fine conductivity, high transmittance and good mechanical flexibility. ${ }^{29,30}$ However, to the best of our knowledge, they are rarely 
reported in flexible PeLEDs and PQD LEDs. In 2016, Bade et al. reported printed PeLEDs based on carbon nanotube anodes and $\mathrm{Ag}$ nanowire cathodes, which possessed the advantages of flexibility and roll-to-roll processability. ${ }^{31}$ However, the devices showed relatively low current efficiency (CE) of $0.6 \mathrm{~cd} \mathrm{~A}^{-1}$ and external quantum efficiency (EQE) of $1.1 \%$ together with low luminance $(L)$ of $200 \mathrm{~cd} \mathrm{~m}^{-2}$. Zhong et al. first reported highly flexible $\mathrm{CH}_{3} \mathrm{NH}_{3} \mathrm{PbBr}_{3}\left(\mathrm{MAPbBr}_{3}\right)$ PQD LEDs on the AgNW/ polymer composite anodes with the maximum current and power efficiencies of $10.4 \mathrm{~cd} \mathrm{~A}^{-1}$ and $8.1 \mathrm{~lm} \mathrm{~W}^{-1}$, respectively. ${ }^{32}$ Lee et al. fabricated flexible organic/inorganic hybrid PeLEDs on a graphene anode and obtained high CE of $18.0 \mathrm{~cd} \mathrm{~A}^{-1} \cdot{ }^{33}$ It is noteworthy that this is the first work of the successful application of graphene in bulk perovskite LEDs, demonstrating that the graphene anode is also suitable for perovskite optoelectronic devices. Even so, to the best of our knowledge, PQD LEDs using graphene as electrodes have not been reported despite the huge potential for applications in next-generation flexible highdefinition displays and high-quality lighting sources.

During continuous applications of a graphene electrode in optoelectronic devices, some of its intrinsic shortcomings such as high sheet resistance, ${ }^{34}$ low work function, ${ }^{35}$ and surface hydrophobicity ${ }^{36}$ have been found to negatively affect the device performances, and these problems need to be resolved to realize excellent electroluminescence (EL) performances. A p-doping treatment on graphene is considered as a useful approach to decrease the sheet resistance and increase the work function of graphene. ${ }^{37-39}$ In addition, we proposed an interfacial modification method of graphene with an ultrathin insulated poly(sodium 4-styrenesulfonate) film, with which we significantly improved the performances of OLEDs and OSCs by reducing the hole injection barrier from graphene to the PEDOT:PSS hole transport layer (HTL). ${ }^{\mathbf{4 0 , 4 1}}$ To overcome the surface hydrophobicity problem, a conventional UV-ozone approach is used to treat the graphene's surface to achieve improved wettability, but this method usually breaks the pristine atomic ordering of graphene and leads to significantly enlarged sheet resistance by changing $\mathrm{sp}^{2}$-bonded carbons to $\mathrm{sp}^{3}$ carbons. An alternative method is the modification of $\operatorname{poly}(3,4-$ ethylenedioxythiophene):poly(styrenesulfonate) (PEDOT:PSS, one of the most widely used commercial hole conductive materials) with IPA or zonyl to improve the wettability of hydrophobic graphene electrodes. ${ }^{42,43}$ The modification of PEDOT:PSS with the above-mentioned organic solvents or nonionic surfactants not only improves the wettability of graphene and forms a uniform film on the graphene surface, but also increases the conductivity of PEDOT:PSS by several orders of magnitude. ${ }^{44}$ More importantly, the PEDOT:PSS coating can sufficiently reduce the graphene electrode's sheet resistance and simultaneously improve its work function via forming $\mathrm{p}$ doped graphene, ${ }^{\mathbf{4 5}, 46}$ which is more suitable for device applications as an anode.

Herein, we reported the first application of graphene into PQD LEDs as an anode and we used small-molecule nonionic surfactant Triton X-100 and DMSO to modify PEDOT:PSS. The addition of Triton X-100 and DMSO into PEDOT:PSS not only improved the wettability of the graphene surface and the sequent film quality containing PEDOT:PSS and poly[ $N, N^{\prime}$-bis(4butylphenyl)- $N, N^{\prime}$-bis(phenyl)-benzidine] (poly-TPD), but also reduced the dissolution of toluene (PQD solvent) to the bottom poly-TPD and PEDOT:PSS films. More importantly, the synergistic effect of Triton X-100 and DMSO altered the PEDOT:PSS morphology from a coiled structure to a nanofibril conductive network, sufficiently enhancing the electrical conductivity of PEDOT:PSS. With this modification strategy, green PQD LEDs with $\mathrm{MAPbBr}_{3}$ emission layers were successfully fabricated on graphene anodes, with 3.7- and 4.4-fold enhancements in luminance and current efficiency, respectively, compared to those of the counterpart without PEDOT:PSS modification. The best EL performance of our green PQD LEDs using graphene anodes is very close to that of the device with an ITO anode.

\section{Experimental}

\subsection{Materials}

The monolayer graphene films were purchased from Hangzhou Gelanfeng Nanotechnology Co. Ltd., which were synthesized on $\mathrm{Cu}$ foils with chemical vapor deposition (CVD) approach. Patterned indium-tin oxide (ITO) glasses with a sheet resistance of $7 \Omega \mathrm{sq}^{-1}$ were purchased from Hua Yu United Technology Co. Ltd. Polymethylmethacrylate (PMMA) with a molecular weight of 30000000 was dissolved in anisole with 6 wt $\%$ concentration. PEDOT:PSS (Clevios P VP AI 4083) was purchased from Heraeus Materials Technology Co. Ltd. Poly-TPD and TPBi were purchased from Lumtec Tech. Corp. $\mathrm{PbBr}_{2}$ and $\mathrm{MABr}$ were purchased from Shanghai MaterWin New Materials Co. Ltd. Oleic acid, octylamine, Triton X-100, DMSO, DMF and other solvents were purchased from Sigma-Aldrich. All materials were used as obtained without further purification.

\subsection{Transfer of graphene electrode}

We used a conventional wet transfer process to transfer graphene. First, PMMA anisole solution was spin-coated onto the graphene-covered copper foil substrate, forming a $\sim 550 \mathrm{~nm}$ protective layer after heating to $120{ }^{\circ} \mathrm{C}$ and annealing for $10 \mathrm{~min}$. Then, the $\mathrm{Cu} /$ graphene/PMMA sheet was placed into $0.1 \mathrm{~mol}\left(\mathrm{NH}_{4}\right)_{2} \mathrm{~S}_{2} \mathrm{O}_{8}$ solution to slowly etch the copper foil. After $10 \mathrm{~h}$, graphene was transferred into deionized water for cleaning $\left(\mathrm{NH}_{4}\right)_{2} \mathrm{~S}_{2} \mathrm{O}_{8}$ and $\mathrm{Cu}^{2+}$, and this step was repeated several times. Next, graphene/PMMA was transferred onto the target substrate by a high-temperature thermal annealing process to remove residual water. Finally, the substrate/graphene/PMMA was placed into boiling acetone to remove residual PMMA. Similarly, this step was repeated several times to eventually obtain the MLG electrode.

\subsection{Preparation of modified PEDOT:PSS films}

The PEDOT:PSS aqueous solution was filtered using a filter ( $0.45 \mu \mathrm{m}$ pore size) prior to use. Then, different amounts of Triton X-100 and DMSO were added into the PEDOT:PSS solution, followed by a six-hour magnetic stirring process for fully mixing of the above-mentioned raw materials. After a 5 min UV ozone treatment to the graphene sheets, the pristine and 
modified PEDOT:PSS solutions were spin-coated onto graphene anodes at $2000 \mathrm{rpm}$ for $45 \mathrm{~s}$. The resulting films were thermally annealed at $120{ }^{\circ} \mathrm{C}$ for $30 \mathrm{~min}$ in air.

\subsection{Characterization of graphene electrode and PEDOT:PSS solution}

The Raman spectra of graphene were measured using a Raman microscope (InVia, Renishaw). The sheet resistance of graphene and conductivity of PEDOT:PSS films were measured with a four-point probe (RTS-9, China). The surface topographies of graphene and graphene/PEDOT:PSS were characterized using an optical microscope. The transmissivities were measured using an ultraviolet-visible spectrophotometer (Shimadzu, UV3600). The surface morphologies of various HTL films were investigated using an atomic force microscope (BRUKER Dimension icon).

\subsection{Synthesis of $\mathrm{MAPbBr}_{3}$ PQD solution}

To prepare $\mathrm{MAPbBr}_{3} \mathrm{PQD}$ solution, $0.40 \mathrm{mmol}$ of $\mathrm{PbBr}_{2}$ and $0.44 \mathrm{mmol}$ of MABr were dissolved in $2 \mathrm{~mL}$ of DMF under vigorous stirring. Then, $220 \mu \mathrm{L}$ of oleic acid and $30 \mu \mathrm{L}$ of octylamine were added into the solution, followed by continuous stirring for 3 hours to obtain the transparent precursor solution. Following this, $0.25 \mathrm{~mL}$ of precursor solution was injected into $10 \mathrm{~mL}$ of toluene solvent with vigorous stirring, and the colorless mixed solution turned bright yellowish-green immediately. At last, the PQD solution was collected and used for subsequent studies and LED fabrication.

\subsection{Characterization of $\mathrm{MAPbBr}_{3}$ PQDs}

The TEM image of $\mathrm{MAPbBr}_{3}$ PQDs was characterized using a transmission electron microscope (Hitachi, HT7700). The PL spectra of PQDs were tested with an IK series (Kimmon Koha, Japan). The UV-vis absorption spectra of PQD films were obtained using a PerkinElmer Lambda $650 \mathrm{~S}$ spectrophotometer with an excitation wavelength of $425 \mathrm{~nm}$. The crystal structures of PQD film were measured using a Bruker D8 ADVANCE X-ray diffraction equipment.

\subsection{Device fabrication and measurement}

The PEDOT:PSS and mixed PEDOT:PSS solutions were spincoated onto graphene or ITO anode to form a $\sim 40 \mathrm{~nm}$-thick HTL. Then, these PEDOT:PSS-covered samples were annealed at $120{ }^{\circ} \mathrm{C}$ in air for $30 \mathrm{~min}$. After this, they were transferred into an $\mathrm{N}_{2}$ glove box to spin-coat the following poly-TPD HTL layer (at a rotation speed of $1500 \mathrm{rpm}$ for $60 \mathrm{~s}$ and then annealing at $140{ }^{\circ} \mathrm{C}$ for $30 \mathrm{~min}$ ) and $\mathrm{MAPbBr}_{3} \mathrm{PQD}$ emitting layer (at a rotation speed of $2000 \mathrm{rpm}$ for $45 \mathrm{~s}$ and then annealing at $50{ }^{\circ} \mathrm{C}$ for 5 min). Notably, spin-coating of the emitting layer was repeated five times. Finally, all samples were loaded into a high-vacuum chamber $\left(5 \times 10^{-7}\right.$ Torr $)$ for film depositions of TPBi $(45 \mathrm{~nm})$, $\mathrm{LiF}(1 \mathrm{~nm})$ and $\mathrm{Al}(100 \mathrm{~nm})$ as the electron transportation layer, the electron injection layer and the cathode, respectively. The EL spectra and luminance-current density-voltage characteristics were tested using a Keithley 2400 source, a calibrated luminance meter, and a PR-655 spectra scan spectrophotometer (Photo Research) in air without further encapsulation.

\section{Results and discussion}

The graphene anode used in this work was obtained from the chemical vapor deposition-grown monolayer graphene (MLG) and it was transferred via a wet process prior to use, with details presented in the Experimental section. In order to observe the quality of the as-obtained MLG, we measured its Raman spectrum. From the result shown in Fig. 1(a), we clearly observe the $\mathrm{G}$ and 2D peaks of graphene at 1593 and $2687 \mathrm{~cm}^{-1}$, respectively. The weak D peak and the low $\mathrm{G}$ peak-to-2D peak intensity ratio $\left(I_{\mathrm{G}} / I_{2 \mathrm{D}}<0.5\right)$ suggest high crystalline quality of the as-used MLG film. ${ }^{47}$ From Fig. S1(a), $\dagger$ the contact angle (CA) of the pristine graphene remains at $56.1^{\circ}$; thus, it is difficult to form a uniform film by directly spin-coating the PEDOT:PSS aqueous solution onto the graphene electrode. To improve the wettability of the graphene surface and the subsequent film quality, a nonionic surfactant Triton X-100 and DMSO (with chemical structures shown in Fig. 1(b)) were added into the PEDOT:PSS aqueous solution; after sufficient stirring, the mixing solutions with Triton X-100, DMSO or both were dropped onto graphene to test CAs. Compared to large CA of $56.1^{\circ}$, the addition of DMSO into PEDOT:PSS slightly reduced the CA to $49.5^{\circ}$ (Fig. S1(b)†), while the introduction of Triton X-100 obviously reduced the CA to $16.9^{\circ}$ (Fig. $\mathrm{S} 1(\mathrm{c}) \dagger$ ), which is beneficial for the formation of a uniform film on graphene. From the photographs and optical microscopy images of various PEDOT:PSS films shown in Fig. $\mathrm{S} 2, \uparrow$ it can be observed that adding Triton $\mathrm{X}$ 100 significantly improves the film uniformity of PEDOT:PSS. In addition, the effect of doping DMSO and Triton X-100 on the transmittance of PEDOT:PSS was investigated (Fig. 1(c)). Compared to $\sim 96.2 \%$ for pristine MLG over the visible spectral range, the transmittance of PEDOT:PSS-covered graphene reduced to $\sim 95.2 \%$, while the addition of DMSO and Triton X100 into PEDOT:PSS resulted in loss of only $\sim 0.5 \%$ in transmissivity, indicating the negligible effects of the additives on the optical properties of the graphene film.

The sheet resistance is also an important parameter to evaluate an electrode's quality. Fig. 1(d) illustrates the effect of different PEDOT:PSS solutions on the sheet resistance of MLG. The sheet resistance was $680 \Omega \mathrm{sq}^{-1}$ for pristine MLG, while it reduced to $430 \Omega \mathrm{sq}^{-1}$ for PEDOT:PSS-coated MLG, indicating the p-type doping of PEDOT:PSS to graphene. ${ }^{\mathbf{4 0 , 4 1}}$ However, with the simultaneous addition of Triton X-100 and DMSO into the PEDOT:PSS solution, the sheet resistance slightly increased due to the insulated nature of Triton $\mathrm{X}-100$. Here, DMSO is considered to be eventually evaporated from the film after the drying process at $120{ }^{\circ} \mathrm{C} .{ }^{48}$ In order to ensure the effect of adding DMSO and Triton X-100 on the electrical properties of the PEDOT:PSS film, the electrical conductivities of various PEDOT:PSS films were measured, as exhibited in Fig. 1(e). The conductivity of the resulting PEDOT:PSS films is highly dependent on the ratio of PEDOT to PSS and the morphology of the films upon drying. ${ }^{49}$ A pure PEDOT:PSS film (Clevios P VP AI4083) has low conductivity of only $\sim 10^{-5} \mathrm{~S} \mathrm{~cm}^{-1}$. Once DMSO 
(a)

(c)
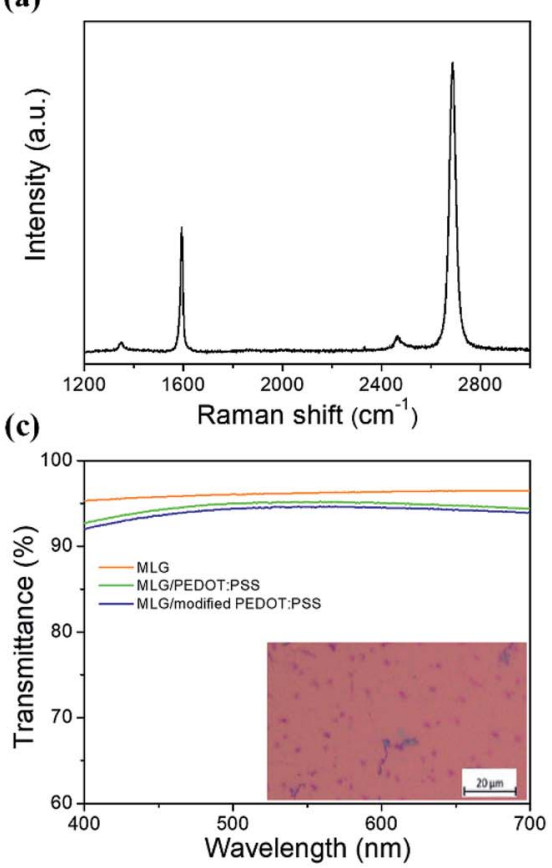

(d)

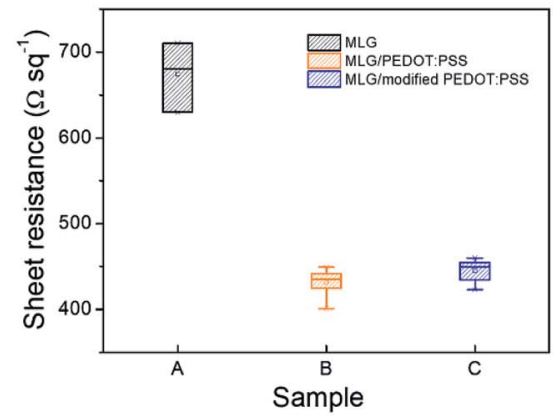

(b)

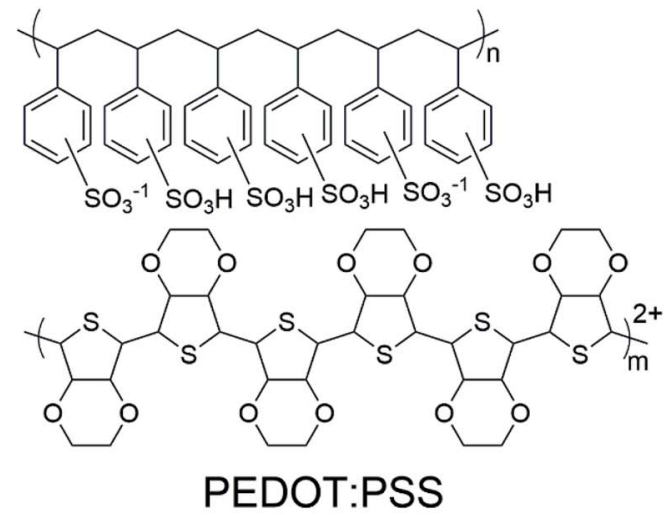<smiles>[H][R]([H])([H])OCCOc1ccc(C(C)(C)CC(C)(C)C)cc1</smiles><smiles>C[Sb](C)=O</smiles>

DMSO (e)

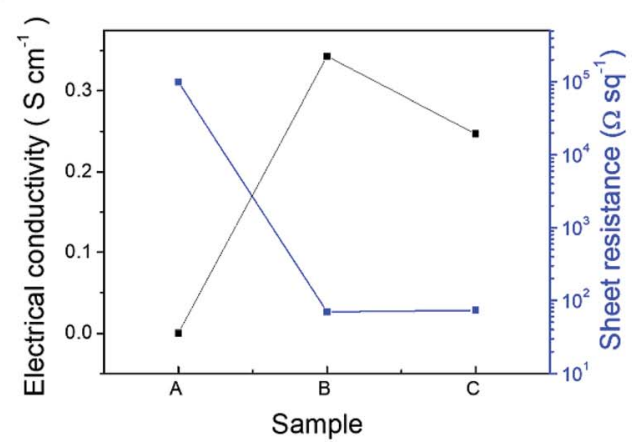

Fig. 1 (a) Raman spectrum of a pristine MLG transferred onto the $\mathrm{SiO}_{2} / \mathrm{Si}$ substrate. (b) Chemical structures of PEDOT:PSS, Triton X-100 and DMSO. (c) Transmittance curves of graphene, graphene/PEDOT:PSS, graphene/modified PEDOT:PSS. All samples are prepared on quartz substrates. (d) Statistics on sheet resistance of pristine MLG, MLG/PEDOT:PSS and MLG/modified PEDOT:PSS. (e) Statistics on conductivity and sheet resistance of PEDOT:PSS (A), two additive-modified PEDOT:PSS (B), Triton X-100-doped PEDOT:PSS (C).

and Triton X-100 are added to the PEDOT:PSS aqueous solution, the interactions induce conformational changes of the PEDOT chains from coiled to linear conformation, resulting in the formation of PEDOT nanofibrils with a good conducting network and better electrical conductivity $\left(0.34 \mathrm{~S} \mathrm{~cm}^{-1}\right)$. The atomic force microscopy (AFM) phase images in Fig. 2 reflect the morphological changes of various PEDOT:PSS films with the increase in Triton X-100 concentration. As can be seen from Fig. 2, the bright and dark regions correspond to the PEDOTrich and PSS-rich zones, respectively. It should be noted that for polymer systems, a lower phase angle (darker region) corresponds to a softer material, while a large phase angle (bright region) represents the rigid conjugated polymer PEDOT with higher conductivity. ${ }^{50}$ From Fig. 2, we can observe that the PEDOT:PSS films show obvious phase separation with the addition of Triton X-100 and DMSO. When the concentration of Triton X-100 changes from 0.01 to $0.05 \mathrm{wt} \%$, the coiled PEDOT structure gradually turns into a nanofibril structure, revealing the formation of a better conductive pathway. However, the continuous increase in Triton X-100 concentration begins to interfere with the conductive PEDOT pathways due to the insulating nature of Triton X-100. As a result, the optimal doping weight ratio for Triton X-100 is about 0.01-0.05 wt\%. To better understand the effects of Triton X-100 and DMSO on the formation of conductive networks, we also observed the AFM phase diagrams of the PEDOT:PSS films with only Triton X-100 or DMSO added. As shown in Fig. S3, $\dagger$ the films with only one additive do not exhibit an obvious nanofibril network, implying that a small fraction of Triton X-100 (0.01-0.05 wt\%) has a synergistic effect with DMSO on forming a conductive network.

We also accidently found that the toluene solvent of PQDs not only re-dissolves the bottom poly-TPD HTL, but also partially removes PEDOT:PSS (please see thickness statistics in 
(a)

Fig. S4(a) $\dagger$ ). Interestingly, the dissolution to both poly-TPD and PEDOT:PSS films is greatly reduced with the doping of DMSO and Triton X-100 (Fig. S4(b)†). About $8 \mathrm{~nm}$ poly-TPD can be reserved on the DMSO- and Triton X-100-modified PEDOT:PSS film, which is in favor of hole injection and transportation in PQD LEDs. In addition, after toluene treatment, the modified PEDOT:PSS/poly-TPD film showed slightly declined surface roughness $\left(R_{\mathrm{q}}\right)$ of $1.40 \mathrm{~nm}$ compared to that of the PEDOT:PSS/ poly-TPD counterpart $(1.70 \mathrm{~nm})($ Fig. $\mathrm{S} 4(\mathrm{c})$ and $(\mathrm{d}) \dagger)$.

Prior to the application of the above-mentioned modified PEDOT:PSS/graphene sheets in PQD LEDs, we synthesized and characterized $\mathrm{MAPbBr}_{3}$ PQDs. Zero-dimensional $\mathrm{MAPbBr}_{3}$ PQDs were synthesized with the conventional ligand-assisted reprecipitation technique; the related details are described in the Experimental section. ${ }^{14}$ The as-prepared DMF precursor solution containing lead bromine $\left(\mathrm{PbBr}_{2}\right)$, methylammonium bromine (MABr), oleic acid and octylamine was injected into the toluene solvent, and the colorless solution immediately turned yellowish-green under vigorous stirring, indicating the formation of $\mathrm{MAPbBr}_{3}$ quantum dots. The diagram of the synthesis process of $\mathrm{MAPbBr}_{3} \mathrm{PQDs}$ and the photograph of the asgenerated PQD solution are shown in Fig. 3(a) and the inset figure of Fig. 3(b), respectively. Under the illumination of a UV light, $\mathrm{MAPbBr}_{3} \mathrm{PQDs}$ emit bright green light with an absolute PLQY of $43 \pm 1 \%$. The transmission electron microscopy (TEM) image in Fig. 3(b) illustrates $\mathrm{MAPbBr}_{3}$ PQDs with an average diameter of $4 \pm 1.5 \mathrm{~nm}$. Fig. 3(c) shows the UV-visible absorption and PL spectra. The PL spectrum exhibits a sharp emission peak at $511 \mathrm{~nm}$ with narrow FWHM of $28 \mathrm{~nm}$. The X-ray diffraction (XRD) patterns in Fig. 3(d) show four diffraction peaks of $14.69^{\circ}, 21.31^{\circ}, 30.29^{\circ}$ and $35.27^{\circ}$, which represent the (100), (110), (200) and (210) crystal planes, respectively, indicating a cubic structure for our products.

For the purpose of anode application, the pristine Fermi level (or called work function) of graphene was low, which remained at $\sim 4.3-4.5 \mathrm{eV}$ in our previous measurements. ${ }^{40,51}$ The low work function and high sheet resistance of graphene will restrain hole injection in LED applications. At the same time, the low conductivity $\left(\sim 10^{-5} \mathrm{~S} \mathrm{~cm}^{-1}\right)$ of PEDOT:PSS also limited hole transport inside the devices. A small amount of Triton X- 
(a)

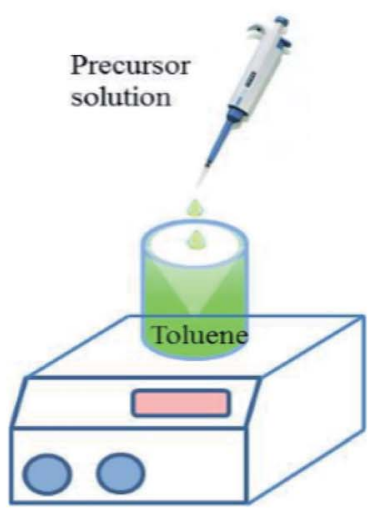

(c)

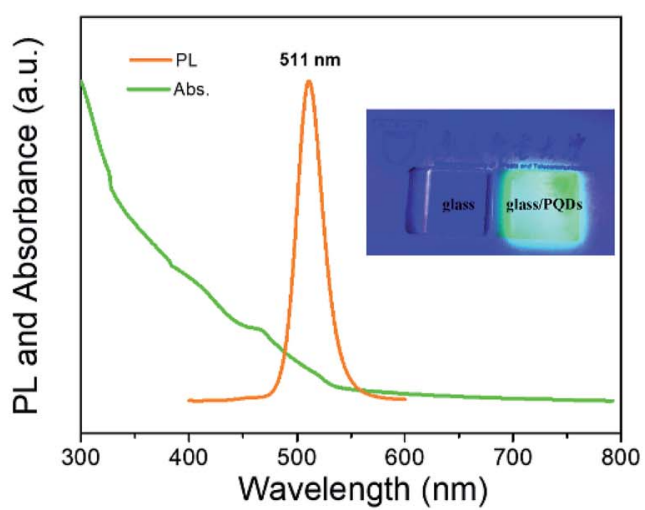

(b)

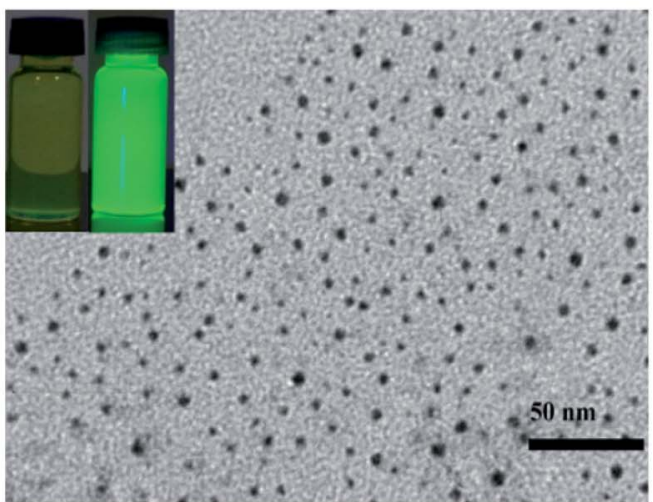

(d)

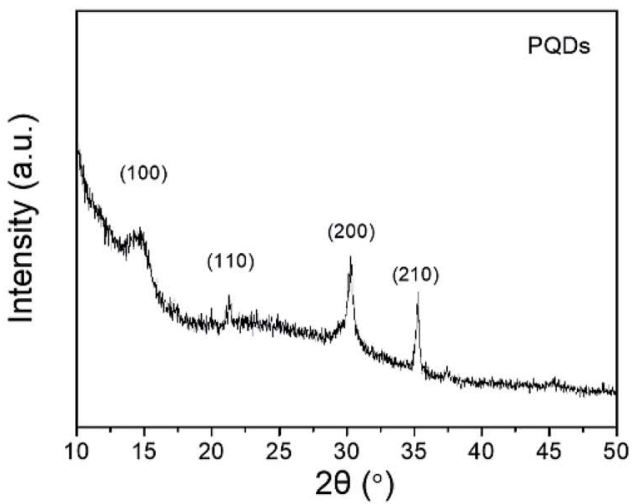

Fig. 3 (a) Synthetic procedure of $M A P b B r_{3} P Q D s$. (b) TEM image of the MAPbBr 3 PQDs. Inset is the $M A P b B r_{3} P Q D s$ in toluene illuminated by a fluorescent lamp and UV light, respectively. (c) PL and absorbance spectra of the $\mathrm{MAPbBr}_{3} \mathrm{PQD}$ film. Inset: Images of glass and glass/PQDs under UV irradiation. (d) XRD patterns of $\mathrm{MAPbBr}_{3}$ PQDs.

100 additive (0.01 wt $\%)$ with different amounts of DMSO was doped into PEDOT:PSS to improve its hole injection/transport ability and film uniformity. The as-used device structure was MLG/PEDOT:PSS (40 nm) : Triton X-100 (0.01 wt\%) : DMSO $(x$ vol\%)/poly-TPD $(45 \mathrm{~nm}) /$ PQDs $(20 \mathrm{~nm}) / \mathrm{TPBi}(45 \mathrm{~nm}) / \mathrm{LiF}(1$ $\mathrm{nm}) / \mathrm{Al}(100 \mathrm{~nm})$, as shown in Fig. 4(a). As illustrated in Fig. S5, $\dagger$ the current density and luminance of the fabricated device obviously improve with DMSO being added into PEDOT:PSS. The luminance increased from $12.6 \mathrm{~cd} \mathrm{~m}^{-2}$ to $100.8,191.6$ and $79.7 \mathrm{~cd} \mathrm{~m}^{-2}$ at $8 \mathrm{~V}$ on increasing the DMSO doping amount to 1 , 2 , and $3 \mathrm{vol} \%$. The turn-on voltage $\left(V_{\text {on }}\right)$ was also reduced significantly from $\sim 4.9$ to $\sim 4.0 \mathrm{~V}$. The ultraviolet photoelectron spectroscopy (UPS) measurements for pristine PEDOT:PSS/MLG and modified PEDOT:PSS/MLG demonstrated that the addition of DMSO and Triton X-100 slightly reduced the work function of PEDOT:PSS from 4.81 to $4.56 \mathrm{eV}$ (Fig. S6 $\dagger$ ), revealing that the increasing EL performances are closely related to the conductivity and film morphology of PEDOT:PSS. This point is well coincident with the observed results of increased injection current densities in all devices with DMSO (Fig. S5(a)†). The optimum doping concentration of DMSO was 2 vol\%. Even so, the luminous efficiency of the best-performing device was still low: only about $0.3 \mathrm{~cd} \mathrm{~A}^{-1}$.
The surfactant Triton X-100 can fully improve the PEDOT:PSS film uniformity and promote the formation of nanofibril-shaped structures; however, introducing more amounts of Triton X-100 into PEDOT:PSS will inevitably induce low conductivity of the PEDOT:PSS film due to the insulating property of Triton X-100, thus generating decreased luminance and CE with increased $V_{\text {on }}$. Thus, in the following part, the doping concentration of Triton X-100 in PEDOT:PSS was adjusted from 0.01 to $0.25 \mathrm{wt} \%$ to find the optimal concentration of Triton X-100. Herein, we simultaneously doped 2 vol\% DMSO into PEDOT:PSS by referring to the experimental results in Fig. S5(a). $\dagger$ From the luminance, current density and CE curves shown in Fig. $\mathrm{S} 7 \dagger$ and the summarized parameters in Table S1, $\dagger$ we ensured that the optimal doping concentration was $0.05 \mathrm{wt} \%$ for Triton X-100. Higher brightness and CE values of $431.2 \mathrm{~cd} \mathrm{~m}^{-2}(8 \mathrm{~V})$ and $2.12 \mathrm{~cd} \mathrm{~A}^{-1}$ were acquired at $0.05 \mathrm{wt} \%$ Triton X-100, which were 8 and 3 times higher than the corresponding values with $0.01 \mathrm{wt} \%$ Triton X-100. To analyze the above-mentioned alteration trend, we fabricated hole-only devices with the structures of MLG/PEDOT:PSS (40 $\mathrm{nm})$ : DMSO (2 vol\%) : Triton X-100 (0.01, 0.05 and $0.25 \mathrm{wt} \%) /$ poly-TPD $(45 \mathrm{~nm}) / \mathrm{MAPbBr}_{3}(20 \mathrm{~nm}) / \mathrm{Ir}(\mathrm{ppz})_{3}(70 \mathrm{~nm}) / \mathrm{Al}(100$ $\mathrm{nm}$ ). As shown in the schematics of the energy levels depicted in 
(a)

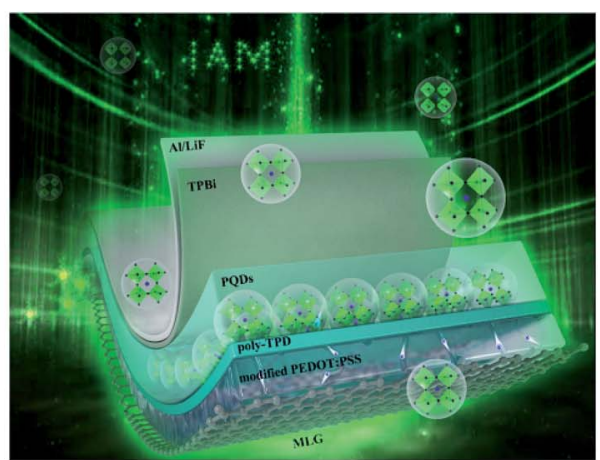

(c)

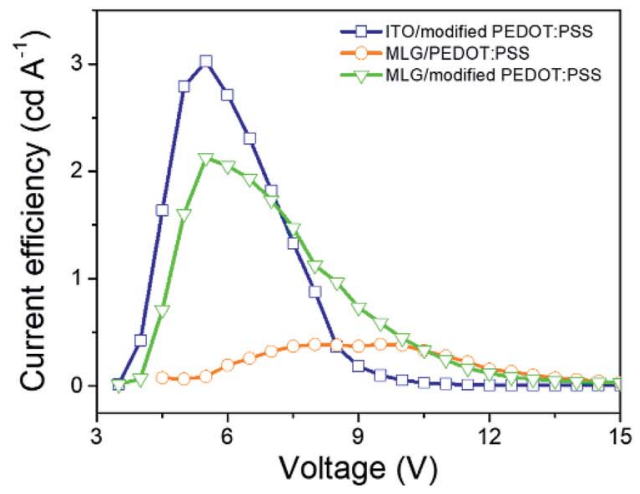

(b)

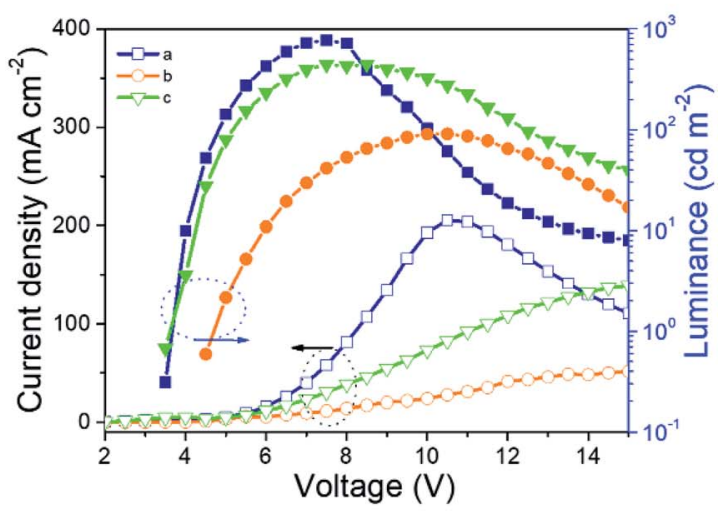

(d)

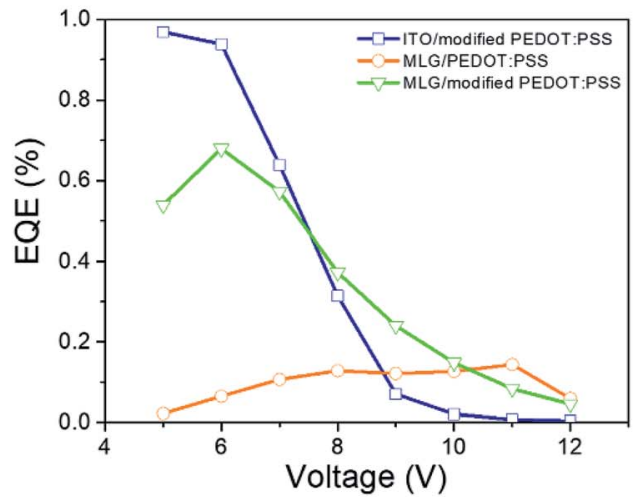

Fig. 4 (a) Diagram of our MAPbBr 3 PQD LEDs fabricated on graphene. (b) Current density and luminance, (c) current efficiency and (d) external quantum efficiency (EQE) curves of our PQD LEDs with graphene and ITO as anodes, respectively. Devices a, b and c represent ITO/ PEDOT:PSS : Triton X-100 (0.05 wt\%) : DMSO (2 vol\%)/poly-TPD/MAPbBr 3 /TPBi/LiF/Al, MLG/PEDOT:PSS/poly-TPD/MAPbBr $3 / \mathrm{TPBi}^{2} \mathrm{LiF} / \mathrm{Al}$ and

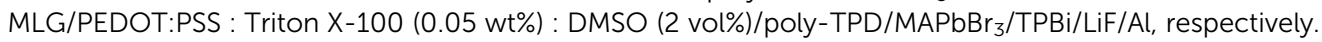

Fig. 5(a), $\operatorname{Ir}(\mathrm{ppz})_{3}$ is used as an effective electron blocking layer owing to its low lowest unoccupied molecular orbital (LUMO) level of $-1.6 \mathrm{eV}$, which can sufficiently block electron injection into the device. Fig. 5(b) compares the injected current densities of the hole-dominated devices with different PEDOT:PSS films, from which the observable increase in current density with a small amount of Triton X-100 added into PEDOT:PSS is mainly due to the formation of the nanofibril-shaped PEDOT conducting network with increasing film hole conductivity. The largest injected hole current is obtained at $0.05 \mathrm{wt} \%$ Triton $\mathrm{X}$ - (a)

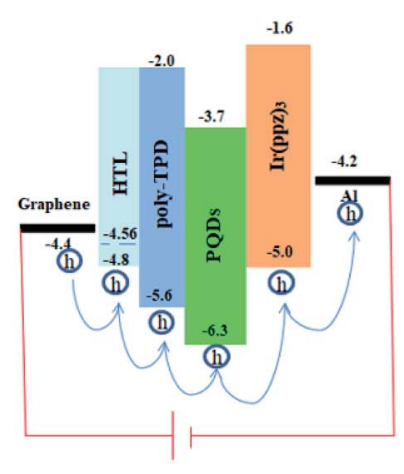

(b)

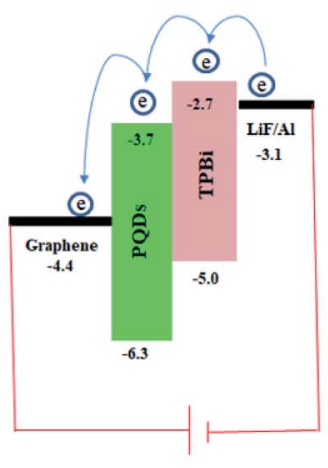

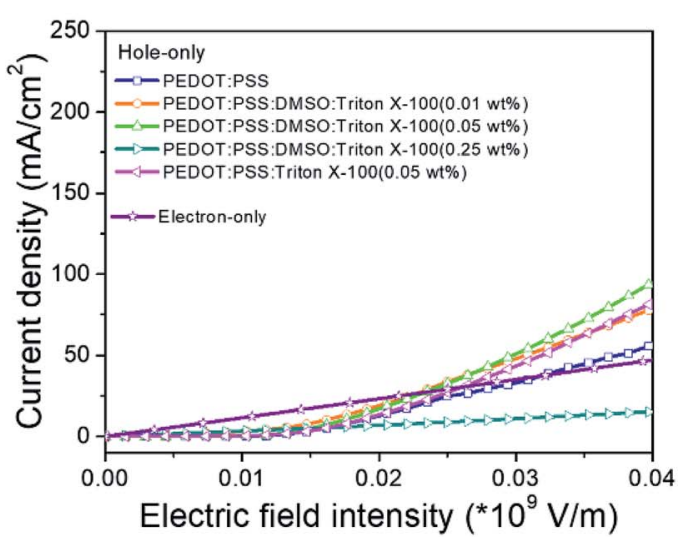

Fig. 5 (a) Energy level diagrams for hole-only and electron-only devices. The HTL is PEDOT:PSS or modified PEDOT:PSS. (b) Current density vs. electric field intensity curves of electron-only device and hole-only devices with various HTLS on MLG anode. The doping ratio of DMSO is 2 vol\%. 
(a)
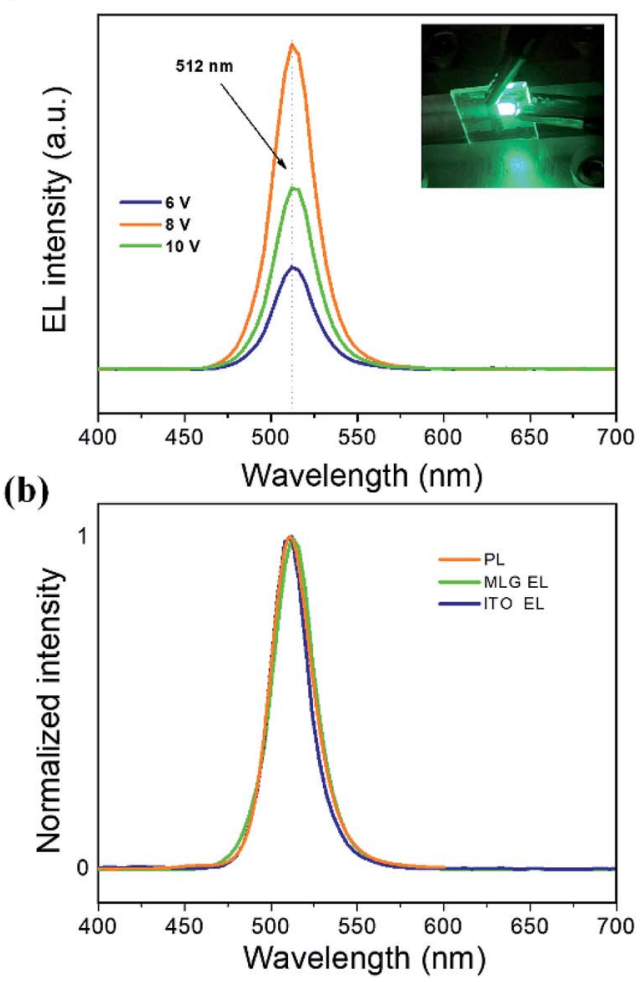

(c)

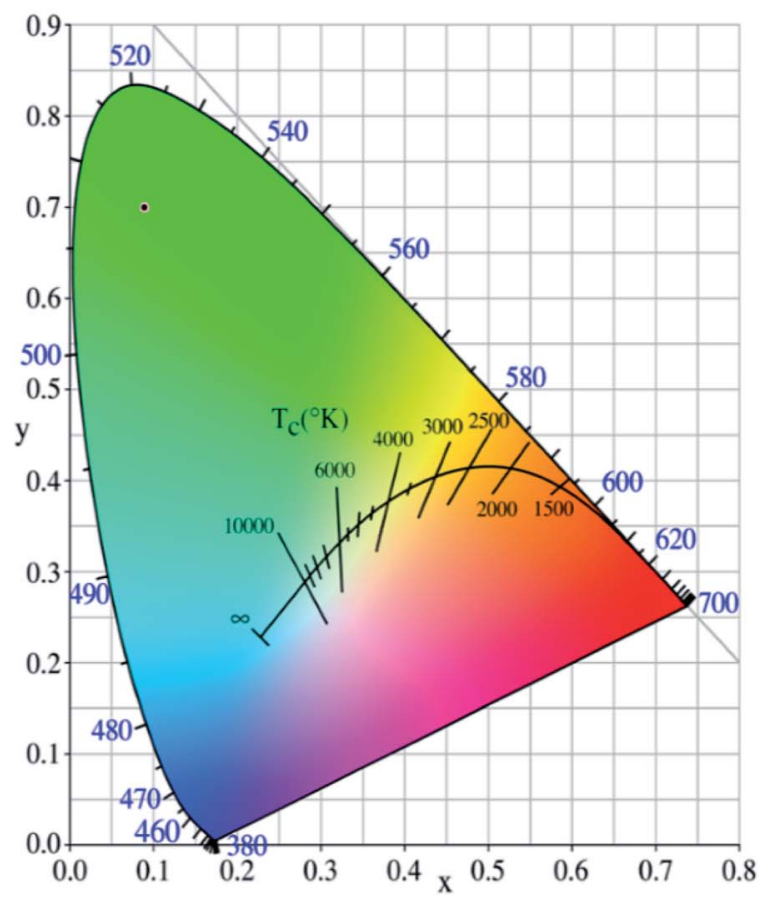

Fig. 6 (a) EL spectra of our graphene-based PQD LED at different voltages. Inset shows a photograph biased at $8 \mathrm{~V}$. (b) PL spectrum of the $\mathrm{MAPbBr}_{3}$ PQDs and EL spectra of PQD LEDs based on graphene and ITO anode. (c) CIE coordinates of our PQD LEDs.

100 and this result is well consistent with the AFM phase image in Fig. 2(c). When more Triton $\mathrm{X}-100$ was doped into PEDOT:PSS, the insulated nature of Triton X-100 led to serious deterioration of film conductivity and significantly declined the injected hole current. Using a moderate Triton X-100 doping ratio of $0.05 \mathrm{wt} \%$ was helpful to improve the balance between holes and electrons, particularly in the electric field intensity range of $0-3 \times 10^{7} \mathrm{~V} \mathrm{~m}^{-1}$ (Fig. 5(b), the electron-only device is fabricated with the structure of MLG/MAPbBr $3(20 \mathrm{~nm}) / \mathrm{TPBi}$ (45 $\mathrm{nm}) / \mathrm{LiF}(1 \mathrm{~nm}) / \mathrm{Al}(100 \mathrm{~nm}))$.

We then compared our graphene-based PQD LEDs with a traditional ITO device, which uses the same structure as the graphene counterpart, that is, ITO/PEDOT:PSS : Triton X-100 $(0.05 \mathrm{wt} \%)$ : DMSO (2 vol\%)/poly-TPD/MAPbBr 3 /TPBi/LiF/Al. Fig. 4(b) displays the current density and luminance data obtained from each type of device. The injected current density of the graphene-based LEDs was still low relative to that of the ITO counterpart, while the luminance and $V_{\text {on }}$ were comparable to those of the ITO device. As shown in Fig. 4(c) and (d), the optimized CE and EQE values are $2.12 \mathrm{~cd} \mathrm{~A}^{-1}$ and $0.67 \%$, respectively, in the graphene-based device, which are very close to those of the ITO contrast one ( $3.0 \mathrm{~cd} \mathrm{~A}^{-1}$ and $0.96 \%$ ); both of these are far larger than those of the control graphene device without any modification ( $0.39 \mathrm{~cd} \mathrm{~A}^{-1}$ and $0.14 \%$ ). In addition, these data demonstrate that good EL performances are observed in our present graphene-based PQD LEDs.

Fig. 6(a) shows the EL spectra of our as-fabricated PQD LEDs with a graphene anode and modified PEDOT:PSS at different voltages. The emission peaks appear at around $512 \mathrm{~nm}$ with narrow FWHM of $\sim 28 \mathrm{~nm}$, and they are consistent with the PQD's PL spectrum with a peak centered at $511 \mathrm{~nm}$ and FWHM of $28 \mathrm{~nm}$ (Fig. 6(b)). The inset of Fig. 6(a) illustrates an emission photograph from a working PQD LED under driving bias of $8 \mathrm{~V}$, showing a pure green light from $\mathrm{MAPbBr}_{3}$, which corresponds to the Commission Internationale de I'Eclairage (CIE) color coordinates of $(0.09,0.70)$, as displayed in Fig. 6(c).

\section{Conclusion}

In summary, in this work, we first developed organic/inorganic hybrid PQD LEDs on graphene electrodes. To overcome pristine hydrophobicity of graphene-induced film nonuniformity, we provided a modification approach to PEDOT:PSS by codoping DMSO and surfactant Triton X-100 into PEDOT:PSS. The modified PEDOT:PSS not only improved the wettability of the graphene anode and the sequent film quality, but also reduced the dissolution of the PQD solvent to the bottom poly-TPD and PEDOT:PSS. More importantly, the synergistic effect of Triton X100 and DMSO altered the PEDOT:PSS morphology from a coiled structure to a nanofibril conductive network, sufficiently enhancing the electrical conductivity of PEDOT:PSS HTL. With this modification strategy, green LEDs with $\mathrm{MAPbBr}_{3}$ PQDs as the light-emitting layers were successfully fabricated on graphene anodes. The luminance and CE values exhibited 3.7- and 4.4-fold enhancements, respectively, over those of the control counterpart without PEDOT:PSS modification; these 
were close to the values of a conventional ITO device. The first graphene electrode-based PQD LED and the film modification strategy offered in this work are expected to shed light on the further design and manufacture of flexible highly efficient PQD display and lighting devices.

\section{Conflicts of interest}

There are no conflicts to declare.

\section{Acknowledgements}

The authors acknowledge financial support from the National Foundation for Science and Technology Development of China (973 project, Grant No. 2015CB932203), the National Key Research and Development Program of China (Grant No. 2017YFB0404501), the National Major Fundamental Research Program of China (Grant No. 91833306), the National Natural Science Foundation of China (Grant No. 61505086, 61675088, 61705111 and 61704091), the Science Fund for Distinguished Young Scholars of Jiangsu Province of China (Grant No. BK20160039), the Natural Science Foundation of Jiangsu Province (Grant No. BM2012010 and BK20170899), the Priority Academic Program Development of Jiangsu Higher Education Institutions (Grant No. YX030002), the Synergetic Innovation Center for Organic Electronics and Information Displays, the National Postdoctoral Program for Innovative Talents (Grant No. BX201700122), and the Open Foundation from Jilin University (Grant No. IOSKL2017KF04 and IOSKL2018KF01), and International Science \& Technology Cooperation Program of Jilin (Grant No. 20190701023GH).

\section{Notes and references}

1 L. C. Schmidt, A. Pertegás, S. González-Carrero, O. Malinkiewicz, S. Agouram, G. Mínguez Espallargas, H. J. Bolink, R. E. Galian and J. Pérez-Prieto, J. Am. Chem. Soc., 2014, 136, 850.

2 M. Y. Leng, Z. W. Chen, Y. Yang, Z. Li, K. Zeng, K. H. Li, G. D. Niu, Y. S. He, Q. C. Zhou and J. Tang, Angew. Chem., Int. Ed., 2016, 55, 1.

3 J. Z. Song, J. H. Li, X. M. Li, L. M. Xu, Y. H. Dong and H. B. Zeng, Adv. Mater., 2015, 27, 7162.

4 M. Mittal, A. Jana, S. Sarkar, P. Mahadevan and S. Sapra, J. Phys. Chem. Lett., 2016, 7, 3270.

5 X. L. Zhang, H. Liu, W. G. Wang, J. B. Zhang, B. Xu, K. L. Karen, Y. J. Zheng, S. Liu, S. M. Chen, K. Wang and X. W. Sun, Adv. Mater., 2017, 29, 1606405.

6 A. Perumal, S. Shendre, M. J. Li, Y. K. E. Tay, V. K. Sharma, S. Chen, Z. H. Wei, Q. Liu, Y. Gao, P. J. S. Buenconsejo, S. T. Tan, C. L. Gan, Q. H. Xiong, T. C. Sum and H. V. Demir, Sci. Rep., 2016, 6, 36733.

7 H. L. Huang, F. C. Zhao, L. G. Liu, F. Zhang, X. G. Wu, L. J. Shi, B. S. Zou, Q. B. Pei and H. Z. Zhong, ACS Appl. Mater. Interfaces, 2015, 7, 28128.
8 S. A. Veldhuis, Y. K. E. Tay, A. Bruno, S. S. H. Dintakurti, S. Bhaumik, S. K. Muduli, M. Li, N. Mathews, T. C. Sum and S. G. Mhaisalkar, Nano Lett., 2017, 17, 7424.

9 F. Liu, Y. H. Zhang, C. Ding, S. Kobayashi, T. Izuishi, N. Nakazawa, T. Toyoda, T. Ohta, S. Hayase, T. Minemoto, K. Yoshino, S. Y. Dai and Q. Shen, ACS Nano, 2017, 11, 10373.

10 B. A. Koscher, J. K. Swabeck, N. D. Bronstein and A. P. Alivisatos, J. Am. Chem. Soc., 2017, 139, 6566.

11 J. Z. Song, J. H. Li, L. M. Xu, J. H. Li, F. J. Zhang, B. Han, Q. S. Shan and H. B. Zeng, Adv. Mater., 2018, 1800764.

12 X. M. Li, Y. Wu, S. L. Zhang, B. Cai, Y. Gu, J. Z. Song and H. B. Zeng, Adv. Funct. Mater., 2016, 26, 2435.

13 F. Zhang, S. Huang, P. Wang, X. M. Chen, S. L. Zhao, Y. P. Dong and H. Z. Zhong, Chem. Mater., 2017, 29, 3793.

14 F. Zhang, H. Z. Zhong, C. Chen, X. G. Wu, X. M. Hu, H. L. Huang, J. B. Han, B. S. Zou and Y. P. Dong, ACS Nano, 2015, 9, 4533.

15 N. K. Kumawat, A. Dey, A. Kumar, S. P. Gopinathan, K. L. Narasimhan and D. Kabra, ACS Appl. Mater. Interfaces, 2015, 7, 13119.

16 Q. C. Zhou, Z. L. Bai, W. G. Lu, Y. T. Wang, B. S. Zou and H. Z. Zhong, Adv. Mater., 2016, 28, 9163.

17 A. Kumar and C. Zhou, ACS Nano, 2010, 4, 11.

18 K. Ellmer, Nat. Photonics, 2012, 6, 809.

19 K. Alzoubi, M. M. Hamasha, S. S. Lu and B. Sammakia, J. Disp. Technol., 2011, 7, 593.

20 S. T. Lee, Z. Q. Gao and L. S. Hung, Appl. Phys. Lett., 1999, 75, 1404.

21 K. Fehse, K. Walzer, K. Leo, W. Lovenich and A. Elschner, Adv. Mater., 2007, 19, 441.

22 W. H. Kim, A. J. Makinen, N. Nikolov, R. Shashidhar, H. Kim and Z. H. Kafafi, Appl. Phys. Lett., 2002, 80, 3844.

23 S. L. Hellstrom, H. W. Lee and Z. Bao, ACS Nano, 2009, 3, 1423.

24 C. Feng, K. Liu, J. S. Wu, L. Liu, J. S. Cheng, Y. Zhang, Y. Sun, Q. Li, S. Fan and K. Jiang, Adv. Funct. Mater., 2010, 20, 885.

25 L. Yao, X. Fang, W. Gu, W. H. Zhai, Y. Wan, X. X. Xie, W. J. Xu, X. D. Pi, G. Z. Ran and G. G. Qin, ACS Appl. Mater. Interfaces, 2017, 9, 24005.

26 N. Li, S. Oida, G. S. Tulevski, S. J. Han, J. B. Hannon, D. K. Sadana and T. C. Chen, Nat. Commun., 2013, 4, 2294.

27 H. Wu, L. Hu, M. W. Rowell, D. Kong, J. J. Cha, J. R. McDonough, J. Zhu, Y. Yang, M. D. McGehee and Y. Cui, Nano Lett., 2010, 10, 4242.

28 Z. B. Yu, Q. W. Zhang, L. Li, Q. Chen, X. F. Niu, J. Liu and Q. B. Pei, Adv. Mater., 2011, 23, 664.

29 T. H. Han, Y. B. Lee, M. R. Choi, S. H. Woo, S. H. Bae, B. H. Hong, J. H. Ahn and T. W. Lee, Nat. Photonics, 2012, 6, 105.

30 D. D. Li, W. Y. Lai, Y. Z. Zhang and W. Huang, Adv. Mater., 2018, 30, 1704738.

31 S. G. R. Bade, J. Q. Li, X. Shan, Y. C. Ling, Y. Tian, T. Dilbeck, T. Besara, T. Geske, H. W. Gao, B. W. Ma, K. Hanson, T. Siegrist, C. Y. Xu and Z. B. Yu, ACS Nano, 2016, 10, 1795. 32 F. C. Zhao, D. Chen, S. Chang, H. L. Huang, K. Tong, C. T. Xiao, S. Y. Chou, H. Z. Zhong and Q. B. Pei, J. Mater. Chem. C, 2017, 5, 531. 
33 H. K. Seo, H. Kim, J. Lee, M. H. Park, S. H. Jeong, Y. H. Kim, S. J. Kwon, T. H. Han, S. Yoo and T. W. Lee, Adv. Mater., 2017, 29, 1605587.

34 J. B. Wu, M. Agrawal, H. A. Becerril, Z. N. Bao, Z. F. Liu, Y. S. Chen and P. Peumans, ACS Nano, 2010, 4, 43.

35 D. Kim, D. Lee, Y. Lee and D. Y. Jeon, Adv. Funct. Mater., 2013, 23, 5049.

36 S. R. Wang, Y. Zhang, N. Abidi and L. Cabrales, Langmuir, 2009, 25, 11078.

37 T. H. Han, S. J. Kwon, N. N. Li, H. K. Seo, W. T. Xu, K. S. Kim and T. W. Lee, Angew. Chem., Int. Ed., 2016, 128, 6305.

38 C. L. Hsu, C. T. Lin, J. H. Huang, C. W. Chu, K. H. Wei and L. J. Li, ACS Nano, 2012, 6, 5031.

39 K. C. Kwon, K. S. Choi and S. Y. Kim, Adv. Funct. Mater., 2012, 22, 4724.

40 Y. F. Zhou, M. Wang, L. Wang, S. L. Liu, S. F. Chen, K. Cao, W. J. Shang, J. Q. Mai, B. M. Zhao, J. Feng, X. H. Lu and W. Huang, Appl. Phys. Lett., 2017, 111, 113302.

41 S. F. Chen, Q. Zhang, W. J. Shang, L. H. Liu, H. T. Yu, S. Zhang, L. L. Deng, M. Wang, M. H. Wang, X. Li, B. X. Mi and W. Huang, Sci. Rep., 2018, 8, 8155.

42 H. Park, Y. Shi and J. Kong, Nanoscale, 2013, 5, 8934.

43 J. H. Chu, D. H. Lee, J. Jo, S. Y. Kim, J. W. Yoo and S. Y. Kwon, Adv. Funct. Mater., 2016, 26, 7234.
44 M. Vosgueritchian, D. J. Lipomi and Z. N. Bao, Adv. Funct. Mater., 2012, 22, 421.

45 X. Z. Zhu, Y. Y. Han, Y. Liu, K. Q. Ruan, M. F. Xu, Z. K. Wang, J. S. Jie and L. S. Liao, Org. Electron., 2013, 14, 3348.

46 Q. Zhang, S. F. Chen, S. Zhang, W. J. Shang, L. H. Liu, M. H. Wang, H. T. Yu, L. L. Deng, G. Q. Qi, L. Y. Wang, S. Y. Han, B. Hu, Q. Kang, Y. J. Liu, M. D. Yi, Y. W. Ma, W. J. Yang, J. Feng, X. G. Liu, H. B. Sun and W. Huang, J. Mater. Chem. C, 2018, 6, 1926.

47 A. C. Ferrari, J. C. Meyer, V. Scardaci, C. Casiraghi, M. Lazzeri, F. Mauri, S. Piscanec, D. Jiang, K. S. Novoselov, S. Roth and A. K. Geim, Phys. Rev. Lett., 2006, 97, 187401.

48 S. Savagatrup, E. Chan, S. M. Renteria-Garcia, A. D. Printz, A. V. Zaretski, T. F. O'Connor, D. Rodriquez, E. Valle and D. J. Lipomi, Adv. Funct. Mater., 2015, 25, 427.

49 X. Crispin, F. L. E. Jakobsson, A. Crispin, P. C. M. Grim, P. Andersson, A. Volodin, C. van Haesendonck, M. Van der Auweraer, W. R. Salaneck and M. Berggren, Chem. Mater., 2006, 18, 4354.

50 Y. Wang, R. Song, Y. Li and J. Shen, Surf. Sci., 2003, 530, 136. 51 M. Wang, H. T. Yu, X. Q. Ma, Y. Yao, L. Wang, L. H. Liu, K. Cao, S. L. Liu, C. Dong, B. M. Zhao, C. Y. Song, S. F. Chen and W. Huang, Opt. Express, 2018, 26, 769. 